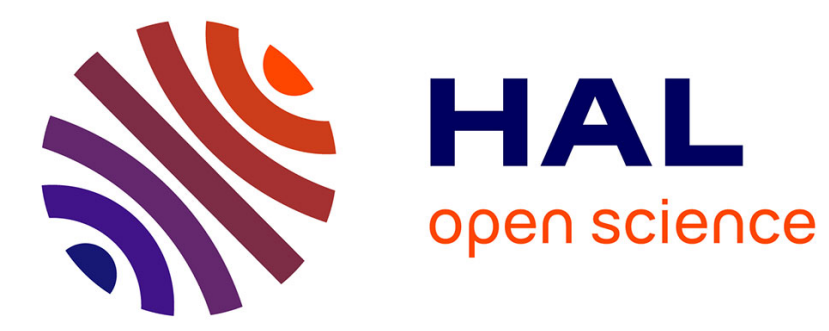

\title{
Intuitive Shape Modeling by Shading Design
}

\author{
Bertrand Kerautret, Xavier Granier, Achille Braquelaire
}

\section{To cite this version:}

Bertrand Kerautret, Xavier Granier, Achille Braquelaire. Intuitive Shape Modeling by Shading Design. 5th International Symposium on Smart Graphics - SG 2005, Aug 2005, Frauenwörth Cloister, Germany, pp.163-174. inria-00000743

\section{HAL Id: inria-00000743 https://hal.inria.fr/inria-00000743}

Submitted on 28 Nov 2005

HAL is a multi-disciplinary open access archive for the deposit and dissemination of scientific research documents, whether they are published or not. The documents may come from teaching and research institutions in France or abroad, or from public or private research centers.
L'archive ouverte pluridisciplinaire HAL, est destinée au dépôt et à la diffusion de documents scientifiques de niveau recherche, publiés ou non, émanant des établissements d'enseignement et de recherche français ou étrangers, des laboratoires publics ou privés. 


\title{
Intuitive Shape Modeling by Shading Design
}

\author{
Bertrand Kerautret $^{1}$, Xavier Granier $^{2}$, and Achille Braquelaire ${ }^{1}$ \\ 1 LaBRI, UMR 5800, Université Bordeaux 1; 351, cours de la Libération \\ 33405 Talence, France \\ 2 IPARLA project (INRIA futurs - LaBRI) \\ \{kerautre, granier, achille\}@labri.fr
}

\begin{abstract}
Shading has a great impact to the human perception of 3D objects. Thus, in order to create or to deform a 3D object, it seems natural to manipulate its perceived shading. This paper presents a new solution for the software implementation of this idea. Our approach is based on the ability of a user to coarsely draw a shading, under different lighting directions. With this intuitive process, users can create or edit a height field (locally or globally), that will correspond to the drawn shading values. Moreover, we present the possibility to edit the shading intensity by means of a specular reflectance model.

Key words: Shape Modeling, Image Based Modeling, Shape From Shading.
\end{abstract}

\section{Introduction}

The shape of a virtual or real $3 \mathrm{D}$ object is mainly perceived by its shading. Our brain is able to infer shape, with only a few ambiguities, from shading alone $[18,1]$. In drawings and paintings artists use shading to suggest shape. Thus, it seems natural to develop an approach based on shading for modeling and
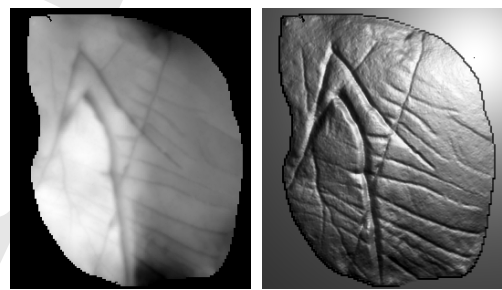
editing $3 \mathrm{D}$ objects.

Using painting and drawing techniques is not new in computer graphics. Sketching approaches (e.g. $[28,11]$ ) have already explored different solutions as fast and intuitive interfaces for creating and editing 3D models. These solutions have shown that a shape can be reconstructed from a set of strokes, lines (e.g. [28]), or from a defined gesture grammar (e.g. [11]).

In our new approach, we want to achieve a different interaction. The user draws what the final object should look like under different lighting conditions. We believe that it is more intuitive to manipulate a height field by its shading rather than directly the height (see figure above). In this modeling approach, the visual feedback is not provided by the direct visualization of the shape, but by a classical 2D drawing/painting. This process is a direct transfer from the visualization of an object in an artist's mind to the drawing. Then, a height field is inferred from these shading images, with a minimal difference of the drawn shading. 
In this paper, we present the following contributions: (i) an intuitive approach for height field modeling, based on drawn shading images, that is not restricted to integrable shadings; (ii) some editing tools, based on shading editing; (iii) some enhancement tools, based on shading editing. Note that we generate only some height fields. However, these kind of models can still be very useful for, material replacement in image [7], or adding relief to planar surfaces [12], to name just a few.

This paper is organized as follows: after an overview of previous work of shape from shading and sketching interfaces, we briefly present our solution for shape recovery from shading images. Afterwards, we develop our approach for the creation of new height fields and editing of existing ones. Finally, we present some results and applications of this work before we conclude.

\section{Previous Work}

Many techniques are used by artists in order to suggest the object's shape, like characteristic lines (or contour lines) or well-designed shading. Since drawing is a familiar task for a lot of people, many researchers have developed some tools inspired by these drawing/painting metaphors, mostly know as "sketching". These approaches can be classified into two categories: the line or stroke based techniques on the one hand, and the gesture based on the other hand.

The most common approach is to infer a $3 \mathrm{D}$ volume from a set of characteristic lines drawn by a user $[6,24,25]$. This can also be done interactively [17, 20]. The ambiguities can be removed by a user-selection of an object list that corresponds to the drawing $[16,8]$. But these approaches are limited to polyhedral models or pre-defined shapes. With other methods, users can design more complex 3D lines, but the final model is still limited to a wireframe one [22]. On the other hand, the stroke-based approaches allow a larger range of possible $3 \mathrm{D}$ lines and 3D curves $[21,2]$. They are mostly limited to illustration since they cannot really reconstruct a full $3 \mathrm{D}$ object.

On the other hand, gesture-based approaches can create a larger range of shapes $[28,11,15]$. Such methods are based on a gesture grammar which is interpreted to define a contour extrusion. These methods allow a global control on the resulting shape, however, a local control is more difficult.

As the shading mostly depends on the normal (i.e., the local variation of the surface), it seems natural to use it for a better control on the resulting shape. Extending the work of Williams [26], Overveld introduced a shape modeling method by painting the surface gradient [23]. Some constraints guarantee that a solution exists, but they disallow the use of classical 2D paint programs and thus reduce the user's freedom. An original approach to edit shape by using shading information was introduced by Rushmeier et al. [19]. This method was used to restore the original shape by using a shading image associated with the region to be edited. This approach is convenient to edit small parts of an existing 3D shape, but it is not adapted to create a new shape without an initial 3D model. Moreover, this approach can suffer from the limitation of the shading intensity contained in only one shading image. 
The first method to reconstruct shape from shading intensity was introduced by Horn in the 70's years [9]. From this first approach, a variety of different methods appeared, but as shown in the survey from Tsang et al. [30], these methods are not robust, especially for the reconstruction from real images. This low robustness represents an important drawback to envisage the shape reconstruction only from the shading information of the drawings.

Even if we suppose a robust shape from shading method, the following points are always problematic:

- A first reason is the well known concave/convex ambiguity $[18,1]$, which becomes particularly apparent when the light source is located in the viewing direction. This ambiguity implies the use of an initial guess in order to recover the shape in such a light source direction.

- When the light source is not in the viewing direction, there is the presence of shadows or inter-reflection. As a consequence, there are image areas which do not contain valid shading information.

- If we consider a drawing, the shading intensity could correspond to an impossible shading image [10]. For example, a compact dark blotch on unit brightness background cannot be a shaded image of a surface with continuous first derivatives.

- A large number of methods first recover normal orientations and then integrate them in order to recover the shape of the final surface. In the presence of noise, the final shape depends on the integration method.

A possible alternative to avoid the problem of a non unique solution of the shape from shading problem is to use more shading information by considering several images associated to different light source directions. This approach, called photometric stereo, was introduced by Woodham [27]. It provides a unique solution for the surface orientation. The results obtained from real data are degraded by noise, shadows, or integrability of the surface normal. There are several approaches that used $N$ light source directions, as for example in [29], but the main drawback is the requirement of a large number of images in order to obtain a precise reconstruction.

\section{Shape Reconstruction from Shading Intensity}

Since our goal is the reconstruction of a shape from one or several shading images which can differ from real shading intensity, we need a really robust reconstruction method. Classical shape from shading or photometric stereo methods are often not robust both in terms of noise and photometric information. For all these reasons, we used a robust approach which shows precise reconstructions with a few number of images [3].

\subsection{Reconstruction by equal height region propagation}

The reconstruction method introduced in $[3,13]$ is based on the equal height region propagation. The strategy of reconstruction consists in considering different

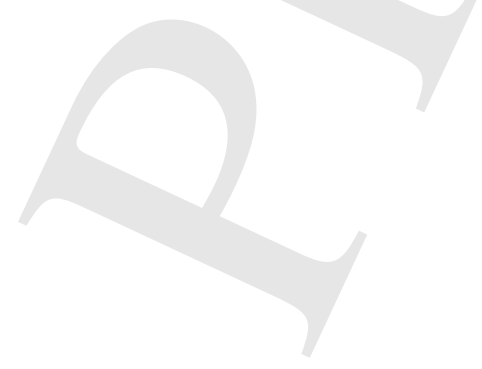


contours of equal height in order to reduce the number of solutions. Generally, it is convenient to define the surface normal orientation by the partial derivatives $p$ and $q$ of the surface that is defined as the bivariate elevation function $Z(x, y)$. Another possibility is to consider the decomposition of the normal vector by the azimuth $(\phi)$ and polar angle $(\theta)$. Now, if we assume that the horizontal component $\left(\phi_{0}\right)$ of the normal vector is known, it can be shown that for a Lambertian surface the solution for the vertical orientation of the normal vector is reduced to the two following solutions:

$$
\theta=\arctan \left(\frac{-\omega \pm \sqrt{-K^{4}+K^{2}+\omega^{2} K^{2}}}{K^{2}-\omega^{2}}\right),
$$

with $K=L_{i} \sqrt{1+p_{s}^{2}+q_{s}^{2}}, \omega=p_{s} \cos \phi+q_{s} \sin \phi$ and $p_{s}, q_{s}$ are associated to the light source direction. $L_{i}$ represents the image intensity.

There are several strategies to select one of the two possible solutions. For example, some continuity constraints can be applied according to the neighborhood, but a more robust approach consists in using more than one shading image in order to overcome this ambiguity.

The reconstruction principle is based on the evolution of patches initialized according to the plane areas of the source images which are orthogonal to the viewer direction. From these initial regions, the reconstruction is made by directly estimating the height of the points that are connected to the patch. In a second step, after this height estimation, the patches are updated by adding all the points for which their new height is included in the equal height interval. This condition is used in order to keep the frontier points of the patch at the same height and thus limiting the possible number of solutions as shown previously.

More details about this height estimation and the patch evolution can be found in [3].

\subsection{Extension to specular models}

Since the height estimation process is not explicitly based on the mathematical expression of the Lambertian surface, this method can be extended to other reflection models such as the extended Lambertian model or the specular/Lambertian model. For the reconstruction of glossy surfaces, we have used the hybrid specular/Lambertian model based on the model of Nayar et al. [14] containing two terms associated to the diffuse and specular component:

$$
I=\rho_{l d} \max \left(0, \cos \left(\theta_{i}\right)\right)+\rho_{l s} e^{\frac{\alpha^{2}}{2 \sigma}}
$$

where $\alpha$ is the angle between the normal and the bisector vector of the incident light source and the viewer direction. $\rho_{l d}$ and $\rho_{l s}$ represent the coefficient of the diffuse and specular component respectively, and $\sigma$ is associated to the roughness surface. Note that the specular spike was note considered.

Generally the Lambertian model associated to a matt surface seems to be well adapted to intuitively draw or complete an existing source image. This model presents the advantage to have an intensity distribution which appears

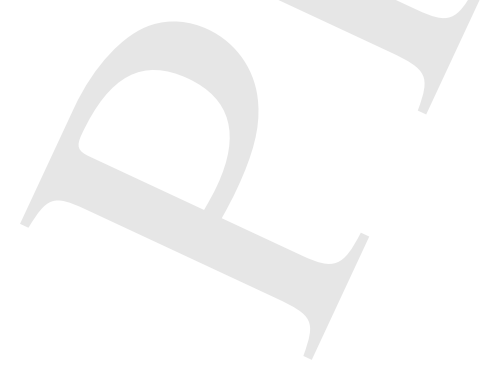




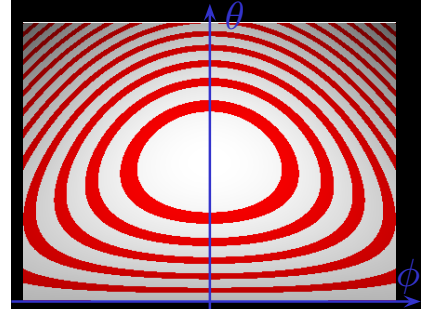

(a)

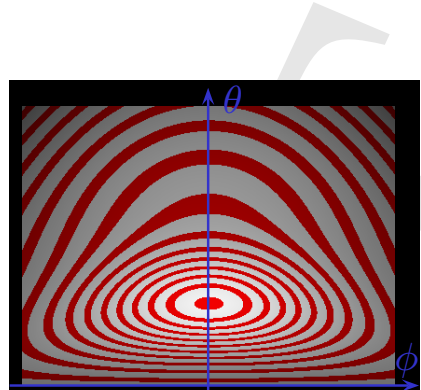

(b)

Fig. 1. Comparison of the reflectance map of a Lambertian (a) and a specular surface (b). The iso-intensity contours are represented in dark.

well adapted according the normal of the surface. Such a distribution can be visualized on the reflectance map which is usually used to represent the distribution of the reflected intensity with respect to the normal orientation $(\phi, \theta)$. An example of a reflectance map associated to a Lambertian surface is represented in Fig. 1-(a). Now, if we consider the reflectance map of glossy/specular models, it appears that the intensity is concentrated in a region associated to the normal orientation near the direction of the light source. This distribution is well visible on the reflectance map in Fig. 1-(b).

From these comparisons of different types of reflectance maps, one can think that it is more difficult for a user to draw fine details with a Lambertian reflectance model since it implies a large precision in using the different shading intensities. On the other hand, with the specular reflectance, fine details could be easier to draw since the intensity gradient is much more important in a particular direction.

\section{Creation, Editing and Enhancement of Height Fields}

Generally, before the final drawing of an object or a character, an artist draws multiple sketches for the different viewing directions and the different lighting conditions. We use this technique for the development of our new modeling and editing approach.

\subsection{Creating the height field}

The first step in the creation of a new height field is the definition of the local and global contours. These can be directly drawn (see Fig. 2-(a)) or extracted from existing images (see Fig. 5-(b)). This step plays an important role since it could help the user to locate the main features in the different drawings associated to different light source directions. These images are the support of the shading definition and contain some discontinuities that we want to keep in the final reconstruction.

In the second step, the user can draw the different shading information associated to several predefined light source directions (see Fig. 2). The orientation 


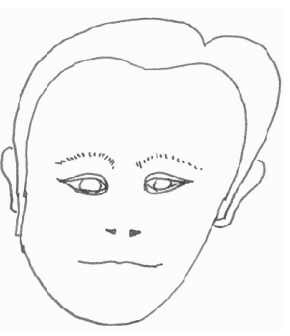

(a)

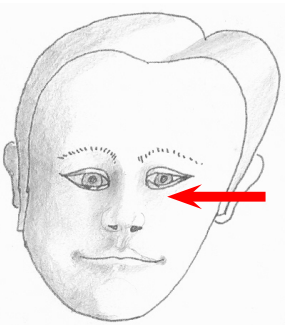

(b)

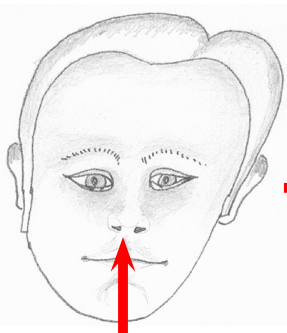

(c)

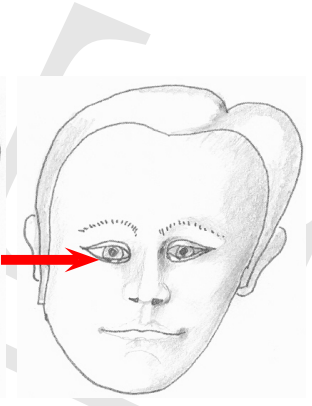

(d)

Fig. 2. Generating source images (b-d) from an initial contour (a). The Images (b-d) are the three of the four images used for the reconstruction. The shading has been drawn according to the light source direction with a vertical orientation of $45^{\circ}$ and a horizontal orientation defined by the arrow.

of the light source direction can be chosen by the user according to different intervals. Note that this step can be done using a classical drawing software, or directly on a real paper by using a scanner, thanks to our robust reconstruction.

The final step is the reconstruction itself. As the reconstruction is done offline, the whole process is not interactive compared to interactive height field reconstruction, like in [23]. But during the whole process, the user has a direct feedback of what the final height field would look like by means of the shading that is currently drawn.

\subsection{Height field enhancement and completion}

We can also use a similar idea to enhance an existing height field or the result of the previous reconstruction. Since a height field can be represented as a grayscaled image, all 2D filters can be directly applied. But, once again, the direct manipulation of height values is less intuitive than the manipulation of shading values. We propose here a three step process. First, we extract some shading images from the existing height field for several light directions. Note that this step is not needed when we already have shading images, like those obtained in the previous section. Then, these images are filtered locally or globally and shape from shading is performed on these new shading images.

We also propose to use a similar approach to add the missing part of an existing object. As for the creation, we can use 2D painting tools to achieve this task. This again is a three step process. We first extract shading images for different light directions. As for the height field enhancement, this step is not needed if we already have the shading images. In the second step, the missing parts can be manually added by using for example the clone-brush of image editing software, or an automatic process like in [5]. We can use all the power of $2 \mathrm{D}$ painting/drawing approaches for completing the missing part of an existing object. The last step is also the final reconstruction.

Compared to mesh-based solution like in [4], we are more restricted (only a $2.5 \mathrm{D}$ object), but our approach gives the complete freedom to the user for

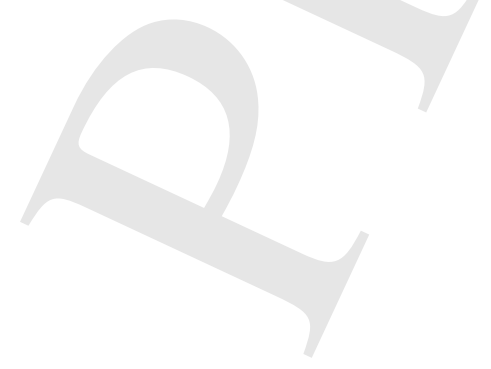




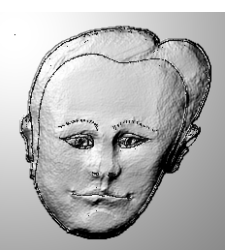

(a)

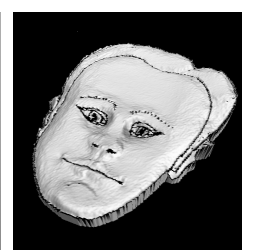

(b)

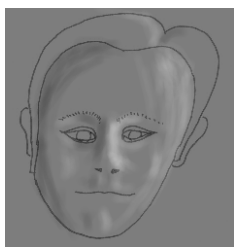

(a)

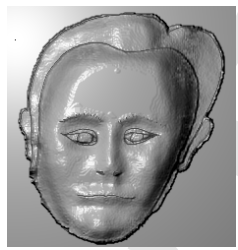

(b)

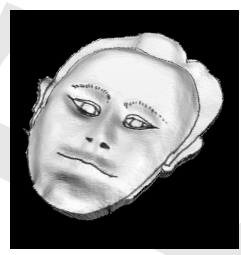

(c)

Fig. 3. Reconstruction results ob- Fig. 4. Reconstruction obtained from other drawtained from the drawings of ings (image (a)). The image (b) was obtained after Fig. 2. The resulting surface is il- rendering the resulting surface and (c) is the 3D lustrated by a rendering and a 3D visualization with the original contour of Fig. 2-(a) visualization. used to create the source image (a).

drawing the missing part, by exploiting all its expertise and knowledge about the edited model. We also believe that it is more intuitive to complete the missing shading than the missing height field. Moreover, it can be done by a non computer experimented user, independently of a specified modeling tool.

\section{Results}

In this section, we present reconstruction results of different types of drawings, the application for height field enhancement, as well as original applications.

\subsection{Reconstruction from drawing patterns}

From the previous drawings of Fig. 2, we applied the reconstruction with the four source images. The reflectance model used for the reconstruction was the Lambertian model for which the albedo was estimated automatically according to the patch initialization on plane areas (i.e., the front and the cheeks of the face). The resulting height field was initially flat and the height interval was adjusted in order to obtained a better visual effect on the resulting surface. Fig. 3(a-b) illustrates the final result of the reconstruction. The image (a) was obtained after rendering the resulting height field with a non-distant light source and by a combined specular/Lambertian model. The initial contour was superimposed with the rendered image. In the same way, the image (b) of the Fig. 3 shows the $3 \mathrm{D}$ visualization of the reconstructed surface.

From the same contour used to create the previous drawings, we have generated four coarse shading images using a classical drawing software. The image (a) of Fig. 4 shows one of the four images used for the reconstruction. These images were first filled by the shading intensity corresponding to plane areas according to the light source direction. Then, different areas were whitened or blackened in an intuitive way. The resulting surface presents a different aspect than the previous example and the final result perfectly fits the initial contour that is superimposed to the resulting surface (Fig. 4-(b-c)).

These two previous examples were generated from the creative imagination of the user. On the other hand, the user can be inspired from an existing image. For example, let us consider the drawing of the Fig. 5-(a). From this image we

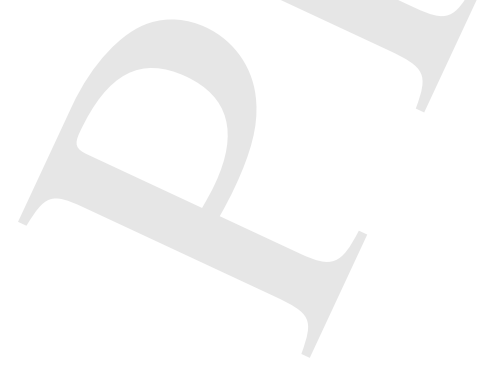




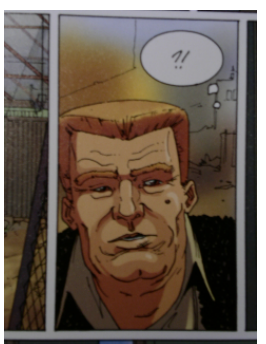

(a)

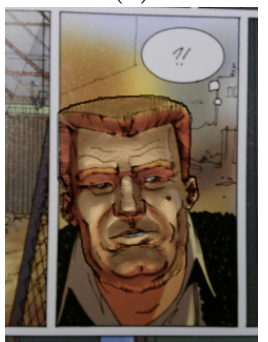

(e)

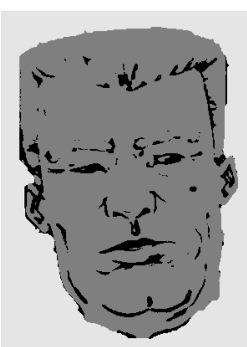

(b)

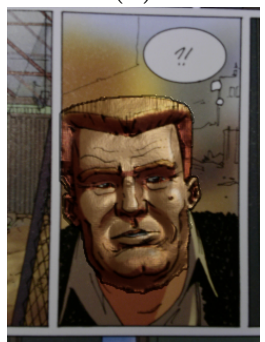

(f)

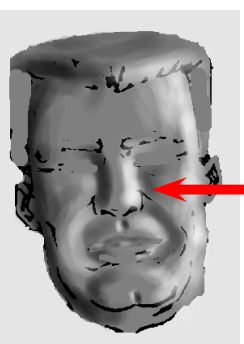

(c)

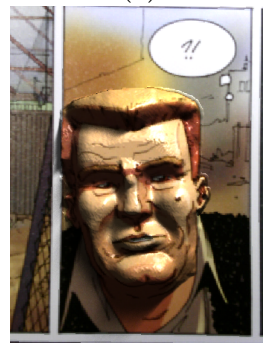

(g)

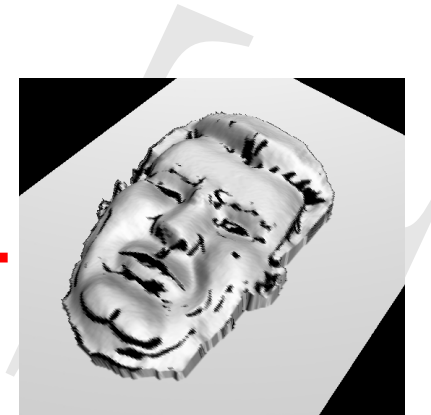

(d)

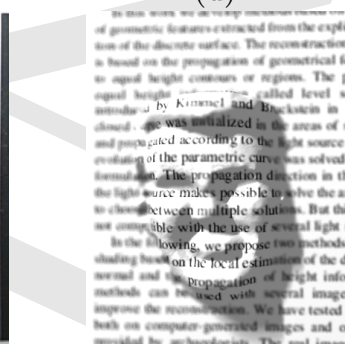

(h)

Fig. 5. Generating sources images (c) from an existing drawing (a-b). The resulting shape is shown in (d). The images (e-h) illustrate the application to image rendering. (c)Brahy/Corbeyran/Braquelaire-Dargaud-Bénélux (s.a. Dargaud-Lombard n.v.)-2004

can first extract the region of interest for which the user wants to recover the depth map. Then, the different contours associated to the main features can be extracted in order to help the user to generate the source images. The image (b) shows the contours extraction obtained from different features as the lip, the nose or the brow. It can be done manually by simply selecting the region of interest and by applying a threshold on this region. Afterwards, the selected region was filled with the intensity associated to a plane area according to the light source direction (with a vertical angle $\theta=45^{\circ}$ ) and the Lambertian reflectance model. As the previous example, the shading intensity was drawn intuitively on the source images (Fig. 5-(c)). These images were drawn using the GimP software and by using the source image superimposed by the transparency of the drawing. When the four images have been drawn, the reconstruction can be applied as described previously. The initial patches were initialized according the plane areas. The image (d) of Fig. 5 shows a $3 \mathrm{D}$ visualization of the resulting height field with the contour image.

\subsection{Application to image rendering}

These reconstruction results can be used for original applications. A possible application is to use the resulting height field in order to improve the original drawing from the initial image. For example, it is possible to obtain new illumination effects by adding a light source including shading areas and shadows defined from the height field. The source image was used as a texture that

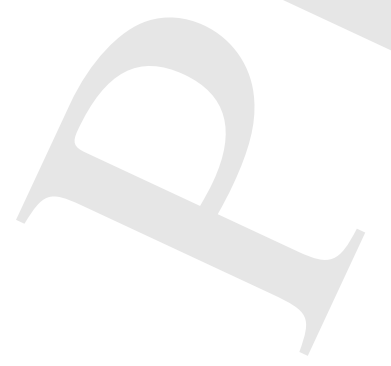




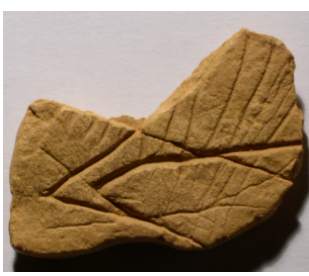

(a)

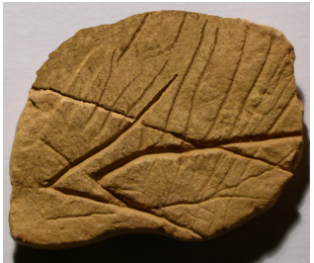

(b)

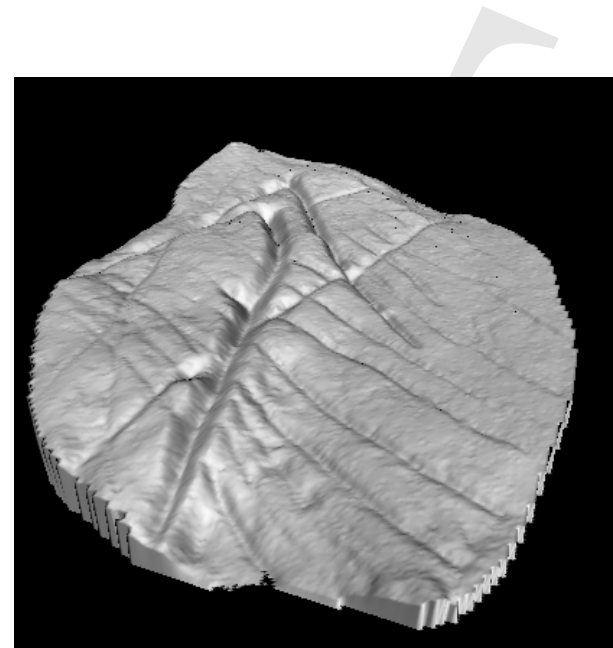

(c)

Fig. 6. Illustration of result obtained from image completions. The image (b) was obtained after completing the image (a). The reconstruction result after the completion of the three source images is shown in (c).

is mapped to the associated height field. Fig. 5 illustrates such obtained new rendering effects. The initial image is shown in Fig. 5-(a) and the new images obtained with different light source directions and different material properties is illustrated in the images (e-g). The resulting images appear still similar to the source images, but they look more realistic than the original. Thanks to the height field which allows to use a non-distant light source rendering, using not only the normals of the surface, but also the height of each point of the surface.

Another application consists in applying blurring effects on the original source image. Since not only the normals of the surface are available, it is possible to simulate a focal length on the original drawing. The image (h) of Fig. 5 illustrates this effect.

\subsection{Image restoration for shape reconstruction}

In order to measure the possibility to use this approach to restore and reconstruct shape, we have selected different old objects. A first example was an archaeological object aged about 35,000 years. This object presents a missing part which was broken (Fig. 6-(a)). In order to reconstruct the object entirely with the missing part, we have completed the image by using the clone brush tools of an image editing software (image (b)). The details of the missing part have been drawn both by continuity and by applying archaeological criteria. Three images were used for the reconstruction. The time to complete the source images was not so much important and was around 20-30 minutes for each image. The resulting surface presented in the Fig. 6-(c) shows as if it was the original object, and there is no difference between the existing and the virtual part of the shape.

Finally, we have applied this main idea to a non-Lambertian object for the reconstruction of an old coin dating from 1818. The coin was used meanwhile

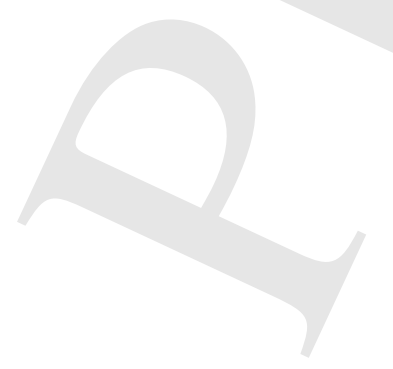




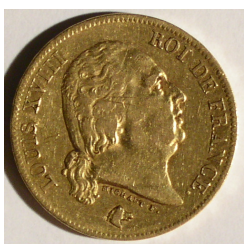

(a)

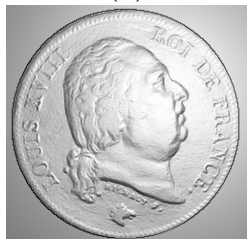

(c)

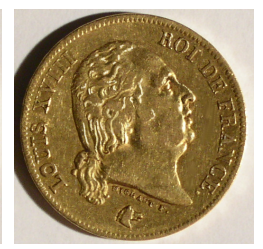

(b)

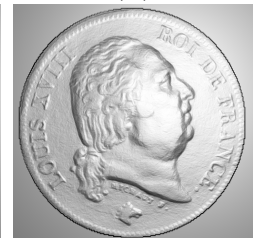

(d)

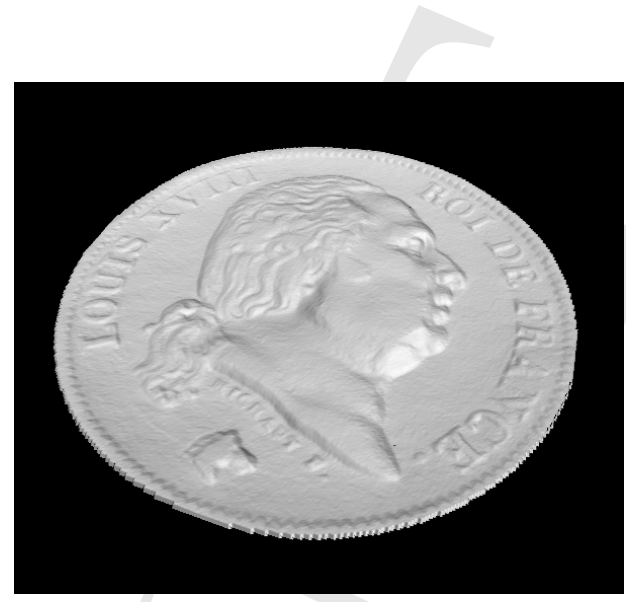

(e)

Fig. 7. Reconstruction results from image enhancement of specular object. The image (a) represents one of the four images of the original object. The image (b) was obtained after adding details on the original image (a). Images (c-e) illustrate the reconstruction obtained from the previous images.

and a number of details were smoothed out or disappeared in comparison to a better conserved coin (Fig. 7-(a)). In order to recover the aspect of the original coin we have edited the four source images used for the reconstruction. It was made by drawing details as the hair, or by accenting the contrast around the letter contours which were smoothed into the original images. From the restored images, we have applied the reconstruction, and all the details performed by the drawing were well reconstructed and visible on the rendering of the resulting height field (Fig. 7-(d)). The different coefficients of the Lambertian/Specular model from Eq. 2 were approximatively estimated by a roughness coefficient $\sigma=0.3$ and by a diffuse/specular constant $\rho_{l s}=0.7, \rho_{l d}=0.3$.

The reconstructions were experimented on an Intel Pentium $31 \mathrm{GHz}$ with $256 \mathrm{Mb}$ of memory. The average times of the different reconstruction processes were included between 2 and less than 5 minutes for a resolution of $500 \times 488$ (resolution of the coin of Fig. 7). The reconstruction process was not optimized since the reconstruction can be done offline after all the drawings were made. Note that the reconstruction process can be adapted to the partial reconstruction of the surface when we consider an interactive surface reconstruction.

\section{Conclusion}

In this paper, we have presented an original method for modeling height fields from shading images. This approach, based on a robust reconstruction method, allows the construction of a shape from shading that are manually drawn by a user. We have presented three possible applications of such an approach, from height field creation, to height field completion and enhancement. The creation or editing process presented here consists always of three steps: users create reference images, and then edit or draw the shading for different lighting directions.

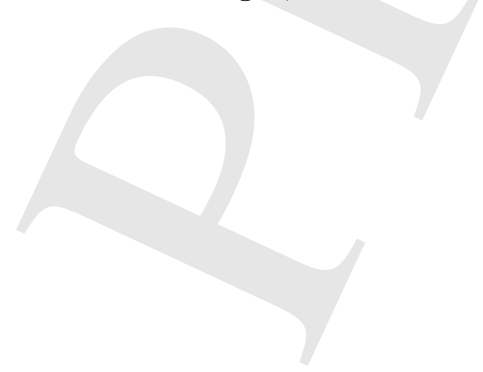


The final step is a shape from shading reconstruction. Even if this work is still limited to height fields, it has shown that working on shading is an intuitive approach for modeling shapes.

This work brings up several fields of reflection. First, we want to create a fully integrated tool that enhances the user-interaction. We also want to experiment more $2 \mathrm{D}$ tools as modeling solutions through shading editing/creation. Finally, we consider to apply this reconstruction to full $3 \mathrm{D}$ object reconstruction and editing.

\section{Acknowledgments.}

We thank Francesco d'Errico from the Institut de Préhistoire et Géologie du Quaternaire for having kindly provided the numerical data of archaeological objects used in this work. We also thank Patrick Reuter for numerous language corrections.

\section{References}

1. Edward H. Adelson and Alex P. Pentland. Perception as Bayesian Inference, chapter The Perception of Shading and Reflectance, pages 409-423. Cambridge University Press, September 1996.

2. David Bourguignon, Marie-Paule Cani, and George Drettakis. Drawing for Illustration and Annotation in 3D. Computer Graphics Forum (Proc. Eurographics 2001), 20(3):114-122, September 2001.

3. Achille Braquelaire and Bertrand Kerautret. Reconstruction of Lambertian Surfaces by Discrete Equal Height Contours and regions propagation. Image and Vision Computing (IVC) Elsevier, 23(2):177-189, February 2005.

4. Levy Bruno. Dual Domain Extrapolation. ACM Trans. Grap. (Proc. SIGGRAPH 2003), 22(3):364-369, July 2003.

5. Iddo Drori, Daniel Cohen-Or, and Hezy Yeshurun. Fragment-based image completion. ACM Trans. Graph. (Proc. SIGGRAPH 2003), 22(3):303-312, July 2003.

6. Lynn Eggli, Ching yao Hsu, Beat D. Brüderlin, and Gershon Elber. Inferring 3D models from freehand sketches and constraints. Computer-Aided Design (JCAD), 29(2):101-112, February 1997.

7. Hui Fang and John C. Hart. Textureshop: texture synthesis as a photograph editing tool. ACM Trans. Graph. (Proc. SIGGRAPH 2004), 23(3):354-359, July 2004.

8. Manuel J. Fonseca, Alfredo Ferreira, and Joaquim A. Jorge. Towards 3D Modeling using Sketches and Retrieval. In Eurographics Workshop on Sketch-Based Interfaces and Modeling, August 2004.

9. Berthold K. P. Horn. Shape from Shading: a Method for Obtaining the Shape of a Smooth Opaque Object from One View. PhD thesis, Departement of Electrical Engineering, MIT, 1970.

10. Berthold K. P. Horn, Richard S. Szeliski, and Alan L. Yuille. Impossible shaded images. IEEE Trans. Pattern Analysis and Machine Intelligence, 15(2):166-170, February 1993.

11. Takeo Igarashi, Satoshi Matsuoka, and Hidehiko Tanaka. Teddy: a sketching interface for 3D freeform design. In Proc. SIGGRAPH '99, pages 409-416. ACM Press/Addison-Wesley Publishing Co., 1999. 
12. Tomomichi Kaneko, Toshiyuki Takahei, Masahiko Inami, Naoki Kawakami, Yasuyuki Yanagida, Taro Maeda, and Susumu Tachi. Detailed Shape Representation with Parallax Mapping. In Proc. the ICAT 2001, pages 205-208, December 2001.

13. Bertrand Kerautret. A Robust Discrete Approach For Shape From Shading and Photometric Stereo. In Proc. ICCVG 2004, 2004.

14. Shree K. Nayar, Katsushi Ikeuchi, and Takeo Kanade. Surface reflection: physical and geometrical perspectives. IEEE Trans. Pattern Analysis and Machine Intelligence, 13(7):611 - 634, 1991.

15. Shigeru Ohwada, Frank Nielsen, Kazuo Nakazawa, and Takeo Igarashi. A Sketching Interface for Modeling the Internal Structures of 3D Shapes. In Proc. Smart Graphics, pages 49-57. Springer-Verlag, July 2003.

16. João P. Pereira, Vasco A. Branco, Joaquim A. Jorge, Nelson F. Silva, Tiago D. Cardoso, and F. Nunes Ferreira. Cascading Recognizers for Ambiguous Calligraphic Interaction. In Eurographics Workshop on Sketch-Based Interfaces and Modeling, August 2004.

17. David Pugh. Designing solid objects using interactive sketch interpretation. In Proc. SI3D '92, pages 117-126. ACM Press, 1992.

18. Vilayanur S. Ramachandran. Perceiving shape from shading. Scientific American, 259(2):76-83, August 1988.

19. Holly Rushmeir, Jose Gomes, Laurent Balmelli, Fausto Bernardi, and Gabriel Taubin. Image-Based Object Editing. In Proc. 3DIM '03, pages 20-28, October 2003.

20. Amit Shesh and Baoquan Chen. SMARTPAPER-An Interactive and User-friendly Sketching System. Computer Graphics Forum (Proc. Eurographics 2004), 24(3), September 2004.

21. Osama Tolba, Julie Dorsey, and Leonard McMillan. Sketching with projective 2D strokes. In Proc. UIST '99, pages 149-157. ACM Press, 1999.

22. Steven Tsang, Ravin Balakrishnan, Karan Singh, and Abhishek Ranjan. A suggestive interface for image guided 3D sketching. In ACM CHI Letters (Conference on Human Factors in Computing Systems), volume 6, pages 591-598.

23. C. W. A. M. van Overveld. Painting gradients: free-form surface design using shading patterns. In Proc. Graphics interface '96, pages 151-158. Canadian Information Processing Society, 1996.

24. P. A. C. Varley, H. Suzuki, and R. R. Martin. Can Machines Interpret Line Drawings? In Eurographics Workshop on Sketch-Based Interfaces and Modeling, August 2004 .

25. P. A. C. Varley, Y. Takahashi, J.Mitani, and H.Suzuki. A Two-Stage Approach for Interpreting Line Drawings of Curved Objects. In Eurographics Workshop on Sketch-Based Interfaces and Modeling, August 2004.

26. Lance Williams. 3D paint. In Proc. SI3D '90, pages 225-233. ACM Press, 1990.

27. Robert J. Woodham. Photometric method for determinating surface orientation from multiple images. Optical Engineering, 19:139-144, 1980.

28. Robert C. Zeleznik, Kenneth P. Herndon, and John F. Hughes. SKETCH: an interface for sketching 3D scenes. In Proc. SIGGRAPH' '96, pages 163-170. ACM Press, July 1996.

29. Ruo Zhang and Mubarak Shah. Iterative shape recovery from multiple images. Image and Vision Computing, pages 801-814, 1997.

30. Ruo Zhang, Ping-Sing Tsai, James E. Cryer, and Mubarak Shah. Shape from shading: A survey. IEEE PAMI, 21(8):690-706, August 1999. 